\title{
Relación entre el índice de área foliar del dosel y el crecimiento de los renovales de coihue y de ciprés de la cordillera
}

\author{
Marina Caselli ${ }^{1,2}{ }^{\otimes} ;$ Gabriel Á. Loguercio $^{2 / 3} ;$ M. Florencia Urretavizcaya ${ }^{1 / 2}$ \\ \& Guillermo E. DefossÉ $E^{1,3 / 4}$ \\ ${ }^{1}$ Consejo Nacional de Investigaciones Científicas y Técnicas (CONICET). ${ }^{2}$ Centro de Investigación y Extensión Forestal \\ Andino Patagónico (CIEFAP). ${ }^{3}$ Universidad Nacional de la Patagonia San Juan Bosco (UNPSJB). ${ }^{4}$ Centro de Investigación \\ Esquel de Montaña y Estepa Patagónicas (CIEMEP).
}

\begin{abstract}
Resumen. La ocupación del espacio de crecimiento del dosel tiene influencia sobre el desarrollo de la regeneración natural. El índice de área foliar (IAF) del dosel es una variable que se puede utilizar en estudios ecológicos y en la toma de decisiones silviculturales para representar el espacio de crecimiento ocupado en un rodal. Es especialmente útil en bosques mixtos, ya que permite captar mejor la complejidad de la estructura forestal que otras variables de densidad. El ciprés de la cordillera (Austrocedrus chilensis) y el coihue (Nothofagus dombeyi) son especies de importancia forestal de los bosques andino-patagónicos, que pueden crecer en formaciones mixtas con potencial productivo. Determinar cuáles son las estructuras forestales necesarias para promover la regeneración implica conocer cómo el espacio de crecimiento que ocupa el dosel afecta el crecimiento de la regeneración avanzada. El objetivo de este estudio fue evaluar la relación entre el IAF del dosel y su distribución entre especies y estratos de altura con el incremento en altura de la regeneración avanzada de ciprés y coihue. El incremento en altura de los renovales muestra una tendencia opuesta al IAF del dosel, aunque esta relación es significativa sólo para coihue, para el cual por debajo de un IAF cercano a 4 favorece el crecimiento de los renovales. A su vez, el incremento en altura de los renovales, en especial del coihue, se ve influenciado negativamente por una participación mayor de coihue en el IAF del dosel. A partir de los resultados se infiere que para promover el crecimiento de la regeneración de ambas especies es necesario mantener, mediante raleos, el IAF por debajo de 4 en los sectores que se requiera promover la regeneración de coihue, y por encima de 4 para la de ciprés de la cordillera.
\end{abstract}

[Palabras clave: incremento en altura, regeneración natural, espacio de crecimiento ocupado, IAF, Austrocedrus chilensis, Nothofagus dombeyi, bosque mixto, manejo forestal]

\begin{abstract}
Aвstract. Coihue and andean cypress saplings growth in relation to canopy leaf area index. The growing space occupancy by the canopy influences the development of natural regeneration. The leaf area index (LAI) is a variable that can be used in ecological studies and in silvicultural decisions process to represent the growing space occupancy in a stand. It is especially useful in mixed forests, as it is better at capturing the complexity of forest structure than other density variables. Andean cypress (Austrocedrus chilensis) and coihue (Nothofagus dombeyi) are species of forest importance of the Andean-Patagonian forests, which can grow in mixed formations with productive potential. Being able to determine which are the target structures of these forests to promote regeneration implies knowing how the canopy growing space occupancy affects the growth of advanced regeneration. The objective of this study was to evaluate the relationship between the canopy LAI and its distribution in the species and height strata with the height increment of advanced regeneration of Andean cypress and coihue. The height increment of the saplings shows an opposite tendency to the LAI of the canopy, although this relationship is significant only for coihue. For this species, the growth of the saplings is favored below a canopy LAI close to 4 . In turn, the height increment, especially for coihue, is negatively influenced by a greater participation of coihue in the canopy LAI. From the results, it is inferred that, in order to stimulate the growth of the regeneration of both species, it is necessary to keep, by thinning, the LAI below 4 in the sectors where it is necessary to promote the regeneration of coihue, and above 4 to promote the Andean cypress regeneration.
\end{abstract}

[Keywords: height increment, natural regeneration, growing space occupancy, LAI, Austrocedrus chilensis, Nothofagus dombeyi, mixed forest, forest management]

Editor asociado: Guillermo Martínez Pastur mcaselli@ciefap.org.ar
Recibido: 7 de Septiembre de 2021

Aceptado: 26 de Noviembre de 2021 


\section{INTRODUCCIÓN}

La ocupación del espacio de crecimiento del dosel, entendido como el conjunto de todos los recursos del sitio utilizados por los árboles (O'Hara and Gersonde 2004), y su distribución entre las especies en el bosque mixto $\left(\mathrm{O}^{\prime}\right.$ Hara 2015), tiene influencia sobre el desarrollo de la regeneración natural (Augspurger 1984; Brokaw 1985; Lieberman et al. 1989; Whitmore 1989; Arturi et al. 2001; Martinez Pastur et al. 2011; Kara and Topaçoðlu 2018). Cuando el espacio de crecimiento está totalmente ocupado, la biomasa del sotobosque está inversamente correlacionada con la biomasa del dosel (Brown and Parker 1994; Valladares et al. 2004). Por ello, cualquier cambio en el dosel está acompañado por cambios, fundamentalmente en el régimen de luz del sotobosque, que afectan a la regeneración natural (Emborg 1998; Kara et al. 2017). La luz que penetra en el dosel y alcanza a la regeneración es el factor más importante que regula el crecimiento de las plantas (Finzi and Canham 2000; Martinez Pastur et al. 2007; Niinemets 2010). Sin embargo, la relación entre el crecimiento de la regeneración y la luz presenta variaciones sin una explicación clara (Drever and Lertzman 2001; Gratzer et al. 2004; Stancioiu and O'Hara 2006a), ya que también otros factores afectan el crecimiento (e.g., la edad y el tamaño de los renuevos), al igual que otros factores del sitio (e.g., la humedad del suelo) (O'Hara et al. 2007; von Arx et al. 2013). A su vez, los cambios en la tolerancia a la sombra con el tamaño del individuo que ocurren en algunas especies añaden más fuentes de variación (Givnish 1988).

Una medida que se ha utilizado para representar el espacio de crecimiento ocupado por los árboles es el índice de área foliar (IAF) (Waring 1983; Long and Smith 1984; O'Hara 1988; O'Hara and Gersonde 2004). Esta variable refleja mejor la ocupación del espacio de crecimiento en bosques mixtos que otras variables de densidad, ya que permite captar de forma adecuada su distribución conjunta, vertical y horizontal (Schuler and Smith 1988). La cantidad total de follaje en el dosel refleja los controles, tanto biológicos como ambientales, que afectan el crecimiento de las plantas (Asner et al. 2003). Se ha sugerido que cada especie tiene un patrón de distribución característico del número de plántulas en el sotobosque en relación al IAF del dosel, relacionado principalmente con la sensibilidad a la luz (Shao 1996). A su vez, las respuestas de las especies arbóreas a las variaciones en las condiciones ambientales que resultan de modificaciones en el dosel son variables (Bazzaz 1979), y estas diferencias interespecíficas tienen implicancias significativas para la dinámica de los bosques (Canham and Marks 1985; Canham 1989; Oliver and Larson 1996). Por ello, para obtener una regeneración vigorosa de composición mixta a través de intervenciones silviculturales se requiere controlar la cantidad, la posición y las especies en el dosel que interceptan la luz y compiten por los recursos del suelo (Lieffers et al. 1999; Stancioiu and O'Hara 2006a). Conocer el espacio de crecimiento ocupado por los distintos componentes del dosel y su relación con el crecimiento de la regeneración permitiría utilizar el IAF como una herramienta para tomar decisiones de manejo forestal en la etapa de regeneración de los rodales.

El ciprés de la cordillera (Austrocedrus chilensis D. Don Pic. Ser. Et Bizzarri) - en adelante, 'ciprés' - y el coihue (Nothofagus dombeyi Mirb. Oerst) son especies de importancia forestal de los bosques andino-patagónicos al este de los Andes (Loguercio et al. 2018), que pueden crecer en formaciones mixtas con potencial productivo (Loguercio 1997; Caselli et al. 2021a). Estos bosques proveen tanto bienes y servicios maderables como no maderables (Hoffmann 1982; Loguercio 1997; Rush and Varela 2019), regulan el clima y el ciclo de agua, fijan el suelo, proporcionan hábitats para el mantenimiento de la biodiversidad (Rush and Varela 2019), mitigan las emisiones de carbono y brindan un gran atractivo paisajístico (Loguercio and Ivancich 2019), enmarcando los centros turísticos y las zonas de recreación de los principales pueblos y ciudades de la Patagonia Norte (Urretavizcaya and Defossé 2013; CIEFAP-MAyDS 2016). Ambas especies tienen requerimientos ecológicos diferentes (Caselli et al. 2019), lo que permite que exista una heterogeneidad de estructuras en su rango de distribución, desde los bosques puros de coihue en sitios húmedos del oeste hasta los puros de ciprés en sitios secos al este, asociándose en rodales mixtos en las zonas intermedias (Loguercio et al. 2018). Sin embargo, en estos bosques se realizan actividades extractivas sin planificación de largo plazo que ponen en riesgo la provisión de bienes y servicios en forma sustentable. A su vez, las experiencias de manejo forestal con continuidad técnica son prácticamente nulas debido, en parte, a la escasez de estudios orientados a la silvicultura en las distintas etapas de desarrollo de los rodales. En este 
sentido, se iniciaron estudios para manejar los bosques mixtos de ciprés y coihue utilizando el índice de área foliar como variable de control de la densidad (Caselli et al. 2020, 2021a), con resultados que muestran la utilidad de esta herramienta para describir la estructura de los rodales y su productividad.

Si bien tradicionalmente ambas especies fueron consideradas intolerantes a la sombra (Donoso 1981), presentan diferencias que dan lugar al desarrollo de bosques mixtos con dos estratos de alturas. El coihue presenta dificultades para regenerar bajo dosel cerrado aparentemente debido a su baja tolerancia a la sombra (Müller-Using and Schlegel 1980; Dezzotti 1996; Amoroso and Larson 2010), por lo que se espera que cuanto mayor sea IAF del dosel menor sea el crecimiento de su regeneración. En cambio, el ciprés es algo más tolerante a la sombra que coihue, lo que le permite persistir mucho más tiempo bajo dosel (Veblen and Lorenz 1987; Veblen 1989; Kitzberger et al. 2000), lo que implica que sería menos sensible a aumentos del IAF del dosel. Si bien existen antecedentes importantes de estudios del efecto de diferentes condiciones de crecimiento sobre los renovales de coihue (Müller-Using and Schlegel 1980; Veblen et al. 1995; Dezzotti 1996; Weinberger and Ramirez 2001; Dezzotti et al. 2004; Soto et al. 2009; Donoso et al. 2013; Pafundi et al. 2016;
Caselli et al. 2019, 2021b) y de ciprés (Dezzotti 1996; Gobbi and Schlichter 1998; Arturi et al. 2001; Letourneau et al. 2004; Urretavizcaya and Defossé 2013, 2019; Pafundi et al. 2014, 2016; Caselli et al. 2019, 2021b), hasta la actualidad no se ha estudiado la relación entre el crecimiento de la regeneración natural y el espacio de crecimiento ocupado por el dosel. Este conocimiento es fundamental para determinar cuáles pueden ser las estructuras objetivo para el manejo forestal de los bosques mixtos de ciprés y coihue en la etapa de regeneración. En este contexto, el objetivo del trabajo fue estudiar en bosques mixtos de ciprés y coihue la relación entre el incremento en altura de la regeneración avanzada de ciprés y coihue, y el espacio de crecimiento ocupado por el dosel, expresado a través del índice de área foliar.

\section{Materiales y Métodos}

\section{Muestreos de campo}

El estudio se realizó en rodales mixtos de ciprés y coihue representativos de este tipo forestal, con regeneración de ambas especies, en la Reserva Forestal Loma del Medio - Río Azul, provincia de Río Negro (entre 41 ${ }^{\circ} 57^{\prime}$ y

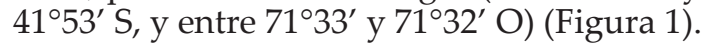
Dicha reserva se asienta sobre suelos del tipo Andisol, cuyo material parental es ceniza

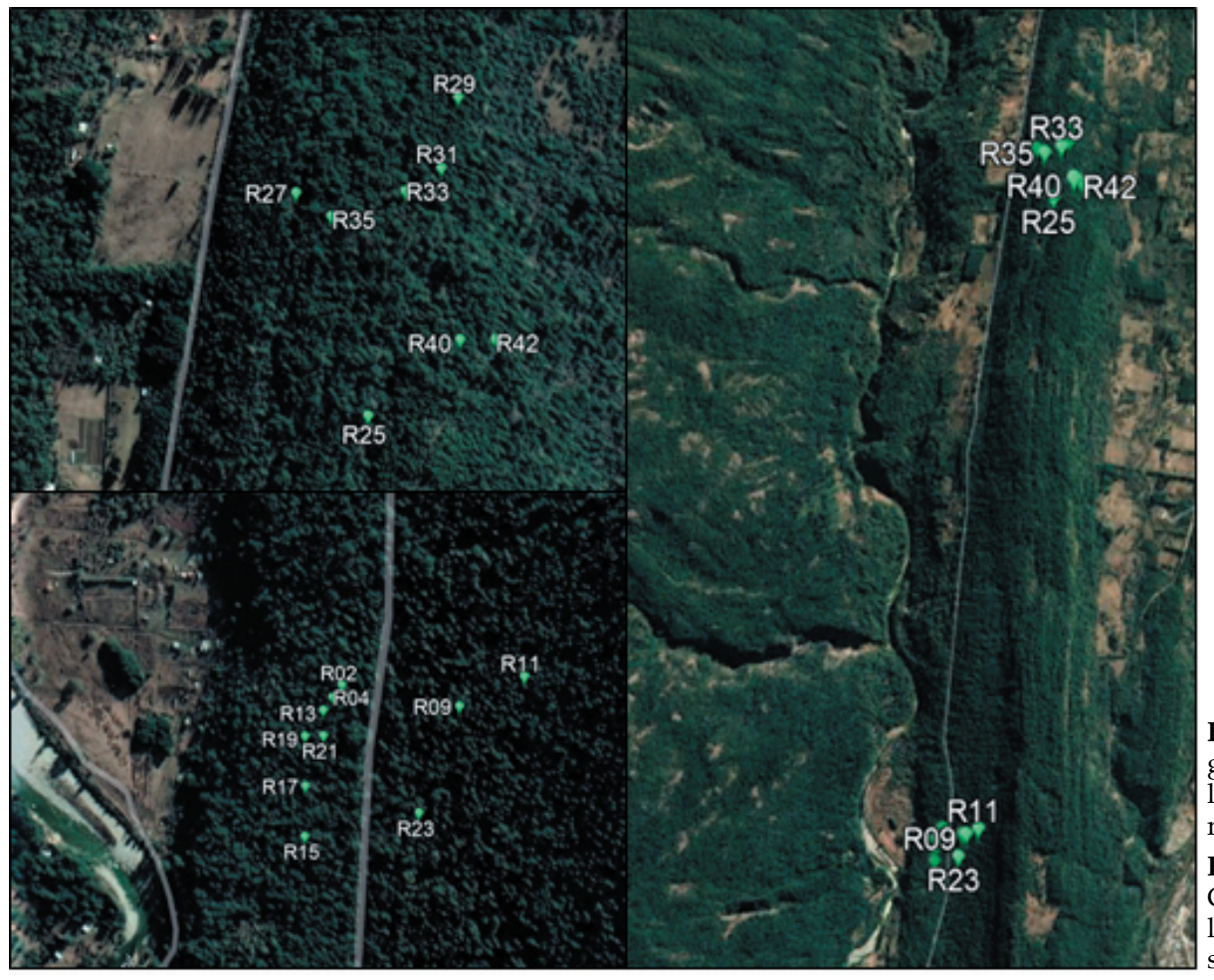

Figura1.Ubicación geografica de los renovales muestreados. Figure Geographic location of the sampled saplings. 
volcánica sobre depósito glaciario; presenta un perfil profundo, buena permeabilidad y alta retención de humedad (Colmet-Daage et al. 1995). La temperatura media anual es 9.3 ${ }^{\circ} \mathrm{C}$ y la precipitación anual es $904 \mathrm{~mm}$, con una ocurrencia marcadamente invernal, con períodos de déficit hídrico en el verano (Arturi et al. 2001). La formación vegetal principal de la reserva corresponde a bosques puros de ciprés $\mathrm{y}$, en menor medida, a bosques mixtos de ciprés-coihue (Chauchard and Barnabá 1986). Estos bosques son formaciones secundarias originadas con posterioridad a los incendios antrópicos de principios del siglo XX (Veblen and Lorenz 1988) y han estado bajo manejo en forma discontinua desde el año 1948 (Chauchard and Barnabá 1986). Las principales cortas en el sitio fueron de saneamiento y tenían como objetivo detener la progresión de la enfermedad 'mal del ciprés'. Con este objetivo, entre 1987 y 1996 se extrajeron $32800 \mathrm{~m}^{3}$ de madera de ciprés, aunque no se logró el saneamiento dadas las características de la enfermedad (Loguercio and Rajchenberg 2004).

Los rodales seleccionados tuvieron una ocupación del espacio de crecimiento de muy alta a muy baja (Figura 2, Tabla 1). Los renovales analizados se eligieron de a pares en cada situación de ocupación por parte del dosel, uno de ciprés y otro de coihue con alturas similares, entre 1.5 y $4 \mathrm{~m}$, ubicados a no más de 3 m entre sí para procurar que tuvieran similares condiciones de crecimiento respecto al dosel arbóreo. En total se muestrearon 20 pares de renovales en distintos rodales (Tabla 1). En torno a los renovales seleccionados se establecieron dos parcelas circulares, una de $1.2 \mathrm{~m}$ de radio $\left(4.5 \mathrm{~m}^{2}\right)$ para determinar la densidad de la regeneración natural teniendo como centro de parcela al renoval, y otra de $10.5 \mathrm{~m}$ de radio $\left(340 \mathrm{~m}^{2}\right)$ para caracterizar la estructura del dosel arbóreo, con su centro entre los dos renovales selectos (Tabla 1, Figura 3).

De cada renoval seleccionado se registró el diámetro a la altura del cuello (DAC) y el diámetro a la altura del pecho (DAP), si correspondía (en dos direcciones perpendiculares), la altura total (m), la altura de inicio de la copa viva $(\mathrm{m})$ y la proporción de la copa viva respecto de la altura total (\%). Luego, de cada renoval se cortó una rodaja del tronco (muestreo destructivo) a la altura del cuello para determinar la edad y el incremento anual en DAC ( $\mathrm{cm} / \mathrm{año})$. Cuando el tamaño del renoval lo permitió, se tomó un

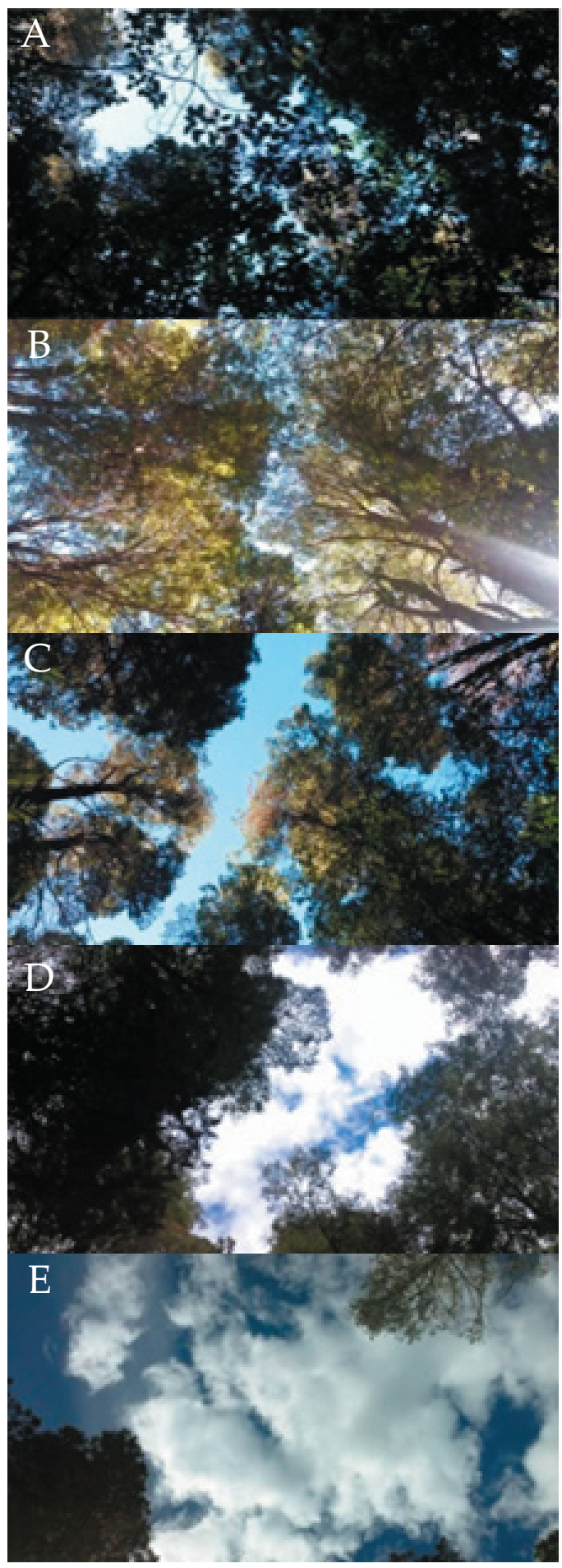

Figura 2. Imágenes de ejemplo del espacio de crecimiento ocupado por el dosel (rango de ocupación) debajo del cual crecían los renovales muestreados. A: parcela R15; B: parcela R-17; C: parcela R-19; D: parcela R-02; E: parcela R-05.

Figure 2. Images of example of the range of canopy growing space occupancy under which the sampled saplings grew. A: plot R-15; B: plot R-17; C: plot R-19; D: plot R-02; E: plot R-05. 
Tabla 1. Atributos de los rodales bajo los cuales crecían los renovales muestreados: Pend: pendiente (\%); Exp: exposición $\left(^{\circ}\right)$; altitud sobre el nivel del mar (m s. n. m.); AB: área basal de coihue y ciprés en los estratos superior e inferior y total del rodal $\left(\mathrm{m}^{2} / \mathrm{ha}\right)$; índice de área foliar del rodal (IAF) de coihue y ciprés en los estratos superior e inferior y total del rodal; Prop IAF coihue (\%): proporción del IAF del rodal ocupada por coihue; Cob: porcentaje de cobertura del dosel.

Table 1. Parameters of the stands under which the sampled saplings grew: Pend: slope (\%); Exp: exposure $\left(^{\circ}\right)$; altitude above sea level (m a. s. 1.); AB: basal area of coihue and cypress in the upper and lower strata and total of the stand $\left(\mathrm{m}^{2} / \mathrm{ha}\right)$; IAF: leaf area index of coihue and cypress in the upper and lower strata and total of the stand; Prop IAF coihue (\%) proportion of the IAF occupied by coihue; Cob: canopy cover \%.

\begin{tabular}{|c|c|c|c|c|c|c|c|c|c|c|c|c|c|c|c|}
\hline \multirow[b]{2}{*}{ Parc. } & \multirow[b]{2}{*}{$\begin{array}{c}\text { Pend } \\
(\%)\end{array}$} & \multirow[b]{2}{*}{$\begin{array}{c}\operatorname{Exp} \\
\left({ }^{\circ}\right)\end{array}$} & \multirow[b]{2}{*}{$\begin{array}{l}\text { Altitud } \\
\text { m s.n. m. }\end{array}$} & \multicolumn{4}{|c|}{ Estrato superior } & \multicolumn{4}{|c|}{ Estrato inferior } & \multirow[b]{2}{*}{$\begin{array}{c}\mathrm{AB} \\
\text { rodal } \\
\left(\mathrm{m}^{2} / \mathrm{ha}\right)\end{array}$} & \multirow[b]{2}{*}{$\begin{array}{l}\text { IAF } \\
\text { rodal }\end{array}$} & \multirow[b]{2}{*}{$\begin{array}{c}\text { Prop } \\
\text { IAF } \\
\text { coihue } \\
(\%)\end{array}$} & \multirow[b]{2}{*}{$\begin{array}{l}\text { Cob } \\
(\%)\end{array}$} \\
\hline & & & & $\begin{array}{c}\mathrm{AB} \\
\text { ciprés } \\
\left(\mathrm{m}^{2} / \mathrm{ha}\right)\end{array}$ & $\begin{array}{l}\text { IAF } \\
\text { ciprés }\end{array}$ & $\begin{array}{c}\mathrm{AB} \\
\text { coihue } \\
\left(\mathrm{m}^{2} / \mathrm{ha}\right)\end{array}$ & $\begin{array}{l}\text { IAF } \\
\text { coihue }\end{array}$ & $\begin{array}{c}\mathrm{AB} \\
\text { ciprés } \\
\left(\mathrm{m}^{2} / \mathrm{ha}\right)\end{array}$ & $\begin{array}{l}\text { IAF } \\
\text { ciprés }\end{array}$ & $\begin{array}{c}\mathrm{AB} \\
\text { coihue } \\
\left(\mathrm{m}^{2} / \mathrm{ha}\right)\end{array}$ & $\begin{array}{l}\text { IAF } \\
\text { coihue }\end{array}$ & & & & \\
\hline R-02 & 17 & 235 & 379 & 16 & 1.6 & 22 & 3.5 & 2 & 0.2 & 2 & 0.2 & 42 & 5.5 & 67 & 69 \\
\hline R-04 & 21 & 275 & 385 & 22 & 2.1 & 36 & 4.6 & 3 & 0.6 & 3 & 0.5 & 64 & 7.9 & 65 & 64 \\
\hline R-05 & 18 & 230 & 379 & 15 & 1.4 & 3 & 0.3 & 1 & 0.5 & 1 & 0.3 & 20 & 2.4 & 25 & 16 \\
\hline $\mathrm{R}-07$ & 27 & 275 & 400 & 23 & 2.1 & 14 & 1.9 & 4 & 0.4 & 1 & 0.2 & 42 & 4.6 & 46 & 76 \\
\hline R-09 & 28 & 245 & 400 & 32 & 3.0 & 6 & 1.5 & 6 & 0.3 & 0 & 0.1 & 44 & 4.9 & 33 & 73 \\
\hline $\mathrm{R}-11$ & 40 & 54 & 341 & 42 & 3.8 & 0 & 0 & 1 & 0.1 & 0 & 0.1 & 42 & 4.0 & 3 & 51 \\
\hline $\mathrm{R}-13$ & 40 & 255 & 363 & 11 & 1.2 & 17 & 2.9 & 6 & 0.5 & 4 & 0.7 & 38 & 5.3 & 68 & 71 \\
\hline R-15 & 34 & 214 & 364 & 10 & 0.9 & 53 & 3.0 & 15 & 1.2 & 1 & 0.3 & 79 & 5.3 & 62 & 85 \\
\hline R-17 & 28 & 283 & 351 & 34 & 3.3 & 9 & 1.8 & 8 & 1.2 & 5 & 1.0 & 56 & 7.4 & 38 & 74 \\
\hline R-19 & 50 & 294 & 350 & 6 & 0.6 & 28 & 3.3 & 5 & 0.5 & 3 & 0.5 & 42 & 4.9 & 78 & 71 \\
\hline R-21 & 25 & 265 & 362 & 5 & 0.6 & 16 & 2.4 & 0 & 0.1 & 5 & 0.8 & 26 & 4.0 & 80 & 79 \\
\hline $\mathrm{R}-23$ & 34 & 260 & 390 & 17 & 1.5 & 0 & 0 & 4 & 0.5 & 0 & 0 & 21 & 2.1 & 0 & 8 \\
\hline R-25 & 7 & 95 & 554 & 13 & 1.3 & 1 & 0.1 & 1 & 0.3 & 4 & 0.7 & 19 & 2.4 & 33 & 36 \\
\hline R-27 & 15 & 144 & 536 & 28 & 2.7 & 0 & 0 & 4 & 0.4 & 1 & 0.1 & 33 & 3.3 & 3 & 49 \\
\hline R-29 & 13 & 55 & 524 & 38 & 3.8 & 3 & 0.4 & 6 & 0.4 & 2 & 0.3 & 49 & 5.0 & 14 & 61 \\
\hline R-31 & 14 & 74 & 533 & 4 & 0.3 & 48 & 4.2 & 5 & 0.5 & 0 & 0 & 57 & 5.0 & 84 & 78 \\
\hline R-33 & 1 & 142 & 555 & 12 & 1.0 & 13 & 0.7 & 9 & 0.8 & 0 & 0.1 & 34 & 2.7 & 30 & 55 \\
\hline R-35 & 25 & 314 & 549 & 17 & 1.5 & 11 & 0.9 & 7 & 0.8 & 1 & 0.4 & 36 & 3.6 & 36 & 45 \\
\hline $\mathrm{R}-40$ & 20 & 230 & 548 & 4 & 0.5 & 11 & 1.4 & 8 & 1.0 & 2 & 0.6 & 25 & 3.4 & 59 & 65 \\
\hline $\mathrm{R}-42$ & 24 & 68 & 536 & 9 & 0.8 & 0 & 0 & 0 & 0.1 & 1 & 0.2 & 10 & 1.1 & 18 & 43 \\
\hline
\end{tabular}

tarugo con un barreno tipo Pressler a la altura del DAC para determinar la edad, en lugar de hacerlo mediante el método destructivo, dejando en estos casos el renoval en pie. Además, se cortó el ápice de cada renoval y se los llevó al laboratorio para determinar el incremento en altura de los últimos 2 años (cm). Para calcular este incremento no se tomó un intervalo de tiempo mayor a fin de no correr el riesgo de que la situación actual de copas no sea representativa del período de tiempo considerado (e.g., por caída de árboles o rotura de ramas). En el caso de ciprés, el incremento anual en altura se determinó mediante la técnica morfo-anatómica de límites interanuales propuesta por Grosfeld (2001). Esta técnica consiste en determinar los límites de crecimiento anual a partir de las características morfológicas de las hojas ubicadas en la zona del límite interanual, que presentan una reducción de su longitud total que se traduce en una disminución en la longitud del entrenudo. En los ápices de los diferentes renovales muestreados se midió la longitud de cada uno de los entrenudos y se realizaron cortes (con tijera de poda) a un lado y otro del entrenudo más pequeño para corroborar el pasaje de un año a otro mediante el conteo del número de anillos de crecimiento. Para contar los anillos se procedió al lijado manual y la utilización de una lupa. Una vez determinado el lugar de cambio de año, se determinó el crecimiento anual del brote. En el caso del coihue, el incremento anual en altura se determinó de manera similar mediante la identificación de las cicatrices de las yemas terminales sobre el tallo (siguiendo a Puntieri et al. [1999] y a Stecconi [2006]) y por conteo de anillos en la base y la parte superior del tallo a la altura de las cicatrices.

En la parcela de densidad de la regeneración (4.5 $\mathrm{m}^{2}$ de superficie) se registró el número de renuevos por especie y clase de altura: $50 \mathrm{~cm} \mathrm{o}$ $2 \mathrm{~m}$ y más de $2 \mathrm{~m}$ hasta $5 \mathrm{~cm}$ de DAP. Los datos de cada renoval pueden verse en la Tabla 2.

Para caracterizar la estructura del dosel, en las parcelas de $340 \mathrm{~m}^{2}$ de superficie, de todos los árboles con DAP mayor a $5 \mathrm{~cm}$ se registró la especie, la posición social (dominantes, 


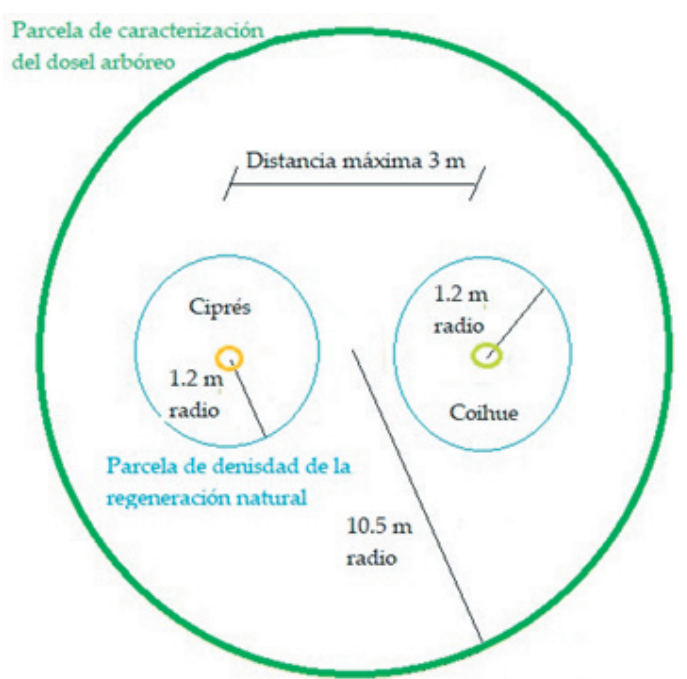

Figura 3. Diagrama de las parcelas de densidad de la regeneración natural (celeste) y de la parcela de caracterización del dosel arbóreo (verde oscuro) en relación con la disposición de los renovales muestreados ciprés (naranja) y coihue (verde claro).

Figure 3. Arrangement of the sampled cypress (green star) and coihue (green circle) saplings, of the plots of natural regeneration density (orange) and of the tree canopy characterization plot (yellow).

codominantes, intermedios y suprimidos, según Oliver and Larson [1996]), el DAP (cm), la altura total $(\mathrm{H}, \mathrm{m})$ y la altura del inicio $(\mathrm{m})$ y mitad (HMC, $\mathrm{m})$ de la copa viva. Además, con un barreno tipo Pressler se tomó un tarugo a la altura del pecho para determinar el área de albura $\left(\mathrm{SABH}, \mathrm{cm}^{2}\right)$ de cada árbol, y con este dato, el área foliar $\left(\mathrm{m}^{2}\right)$. Para esto último se clasificaron los árboles en dos estratos de altura, el estrato superior (compuesto por árboles dominantes y codominantes) y el inferior (compuesto por los intermedios y suprimidos) (Oliver and Larson 1996). Luego se aplicaron las siguientes ecuaciones para determinar el área foliar de cada árbol (Caselli et al. 2020):

Estrato inferior de ciprés: área foliar $=13.1029$ $+0.2665 \mathrm{SABH}-1.5520 \mathrm{HMC}$

Estrato inferior de coihue: área foliar $=0.1344$ $\mathrm{SABH}+5.7549 \mathrm{H} / \mathrm{HMC}$

Estrato superior de ciprés: área foliar $=0.1303$ $\mathrm{SABH}+0.0417 \mathrm{DAP}^{\wedge} 2$

Estrato superior de coihue: área foliar $=$ $0.4708 \mathrm{SABH}-5.9025 \mathrm{DAP}+0.0957 \mathrm{H}^{\wedge} 2$

Con la suma de las áreas foliares de los árboles dividida por la superficie de la parcela se determinó el índice de área foliar (IAF) total, por especie y por estrato de altura (ver Tabla
1). De similar manera se determinó el área basal por hectárea por especie y estrato de altura. También se determinó el porcentaje de cobertura mediante imágenes digitales procesadas con el software libre ImageJ 1.52a (Rasband 2018). Adicionalmente, en cada una de estas parcelas se registró la exposición, la pendiente y la altitud sobre el nivel del mar (Tabla 1).

\section{Análisis estadístico}

Los atributos de los renovales (i.e., edad, DAC, DAP, altura total, altura base de la copa viva, razón de copa viva, incremento medio en DAC, incremento en altura de los últimos 2 años y densidad de renovales por clase de altura) se evaluaron mediante análisis de varianza (ANOVA), con la prueba de Tukey (muestras apareadas) para detectar diferencias significativas entre medias $(\mathrm{P} \leq 0.05)$. Cuando las variables no cumplieron con el supuesto de normalidad se realizaron transformaciones. El supuesto de normalidad se comprobó con la prueba de Shapiro-Wilks. Además, se determinó la correlación entre el IAF del dosel y la edad de los renovales calculando el coeficiente de correlación de Pearson.

Se analizó la relación entre el IAF del dosel (total, por especie y por estrato) y el incremento en altura de los renovales de ambas especies, gráficamente, mediante análisis de regresión lineal y no lineal y mediante análisis de componentes principales (ACP). El ACP se realizó estandarizando los datos y se incluyeron además del IAF, el área basal del dosel (total, por especie y estrato), la altitud, la pendiente y la exposición del sitio. Todos los análisis estadísticos se realizaron con el software Infostat versión 2019 (Di Rienzo et al. 2017).

\section{Resultados}

\section{Atributos de los renovales}

Los renovales de ciprés y coihue presentaron dimensiones similares, se encontraron en condiciones de densidad comparables y no presentaron diferencias significativas de crecimiento. Sin embargo, se diferenciaron en sus edades y en la altura de inicio de la copa viva (Tabla 2).

El incremento en altura de los últimos 2 años de los renovales de coihue se redujo con la edad, mientras que no se observó esta tendencia en ciprés (Figura 4). La edad de los 
Tabla 2. Atributos de los renovales de ciprés y coihue (promedio y error estándar). DAC: diámetro a la altura del cuello; DAP: diámetro a la altura del pecho. La densidad corresponde a la parcela de $4.5 \mathrm{~m}^{2}$ alrededor de cada renoval de ciprés y coihue.

Table 2. Characteristics of cypress and coihue saplings (mean and standard error). DAC: collar diameter; DAP: diameter at breast high. The density corresponds to the $4.5 \mathrm{~m}^{2}$ plot around each cypress and coihue sapling.

\begin{tabular}{|c|c|c|c|c|}
\hline & Ciprés & Coihue & P-valor & $\begin{array}{c}\mathrm{F} \\
\text { (prueba de } \\
\text { Fisher) }\end{array}$ \\
\hline Edad & $19(1.4)$ & $14(0.9)$ & 0.022 & 10.87 \\
\hline $\mathrm{DAC}(\mathrm{cm})$ & $3.5(0.3)$ & $3.2(0.2)$ & 0.501 & 0.46 \\
\hline $\mathrm{DAP}(\mathrm{cm})$ & $1.8(0.2)$ & $1.8(0.2)$ & 0.815 & 0.06 \\
\hline Altura total (m) & $2.9(0.2)$ & $3.1(0.2)$ & 0.403 & 0.71 \\
\hline Altura base de la copa viva (m) & $0.9(0.1)$ & $0.6(0.1)$ & 0.024 & 5.55 \\
\hline Razón de copa viva (\%) & $67.3(4.1)$ & $79.0(4.9)$ & 0.077 & 3.31 \\
\hline Incremento medio en DAC (mm/año) & $1.8(0.2)$ & $2.4(0.2)$ & 0.111 & 2.66 \\
\hline Incremento últimos 2 años en altura $(\mathrm{cm})$ & $28.2(2.9)$ & $42.1(6.6)$ & 0.103 & 2.78 \\
\hline Densidad de ciprés $0.50-2 \mathrm{~m}$ de altura $/ \mathrm{m}^{2}$ & $0.5(0.1)$ & $0.8(0.3)$ & 0.342 & 0.94 \\
\hline Densidad de coihue $0.50-2 \mathrm{~m}$ de altura $/ \mathrm{m}^{2}$ & $0.1(0.06)$ & $0.3(0.1)$ & 0.092 & 2.98 \\
\hline Densidad de ciprés $>2 \mathrm{~m}$ de altura $/ \mathrm{m}^{2}$ & $0.4(0.1)$ & $0.1(0.05)$ & 0.061 & 3.72 \\
\hline Densidad de coihue $>2 \mathrm{~m}$ de altura $/ \mathrm{m}^{2}$ & $0.1(0.03)$ & $0.1(0.04)$ & 0.657 & 0.20 \\
\hline
\end{tabular}

renovales de ciprés no estuvo correlacionada con el IAF del rodal (coeficiente de correlación de Pearson=-0.07, $\mathrm{P}=0.78$ ), mientras que la de coihue mostró una correlación positiva (coeficiente de correlación de Pearson $=0.48$, $\mathrm{P}=0.03)$.

\section{Incremento en altura y su relación con el índice de área foliar del dosel}

El incremento en altura de los renovales de ambas especies se relacionó negativamente con el IAF total del dosel (Figura 5). En coihue,

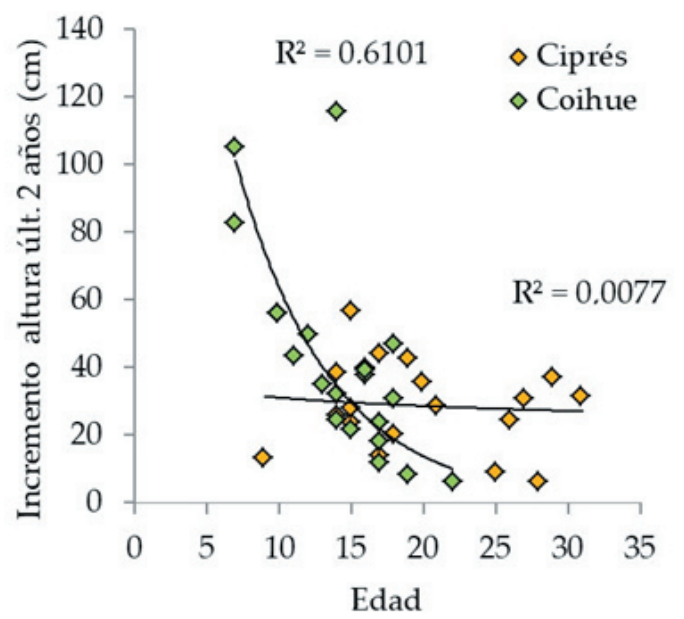

Figura 4. Incremento en altura de renovales de ciprés y coihue de los últimos 2 años en relación con su edad. Las líneas corresponden a líneas de tendencia para las especies, con su respectivo coeficiente de determinación $\left(\mathrm{R}^{2}\right)$.

Figure 4. Height increment of cypress and coihue saplings in the last 2 years in relation to their age. The lines correspond to trend lines for the species, with their respective coefficient of determination $\left(R^{2}\right)$. esta relación fue más notable, especialmente cuando el IAF fue menor a 4. Con un IAF menor a 2.5, el incremento en altura varió

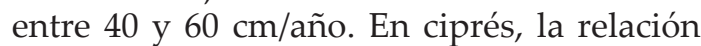
entre el incremento en altura y el IAF del rodal fue menos marcada, pero igualmente mostró una tendencia a un aumento gradual a medida que disminuyó el IAF del rodal.

Al separar el IAF del rodal por especie y estrato de altura se observa que los renovales mostraron mayor crecimiento cuando el IAF de coihue en el rodal fue menor (Figura 6).

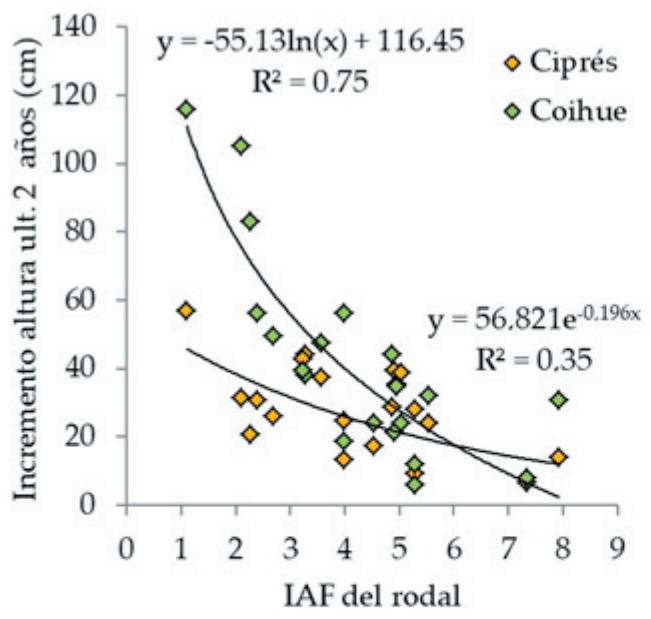

Figura 5. Incremento en altura en los últimos 2 años (cm) para renovales de ciprés y coihue en relación con el índice de área foliar (IAF) del rodal. Las líneas corresponden a líneas de tendencia para cada especie, con su respectivo coeficiente de determinación $\left(\mathrm{R}^{2}\right)$.

Figure 5. Height increment in the last 2 years $(\mathrm{cm})$ for cypress and coihue saplings in relation to the leaf area index (IAF) of the stand. The lines correspond to trend lines for the species, with their respective coefficient of determination $\left(R^{2}\right)$. 
En general, los renovales de ambas especies que mostraron incrementos mayores a $40 \mathrm{~cm}$ en los últimos 2 años pertenecen a parcelas con IAF menor a 4 ó bien con IAF mayor a 4, pero con el estrato superior ocupado en su mayor parte por ciprés (Figura 6). En parcelas con IAF mayor a 4, los incrementos de ciprés y coihue fueron similares. La excepción son las parcelas con el dosel superior ocupado únicamente por ciprés, donde los renovales de coihue mostraron sustancialmente mayor incremento que los de ciprés.

El ACP para los renovales de ciprés y coihue confirma las tendencias observadas. El ACP para los renovales de ciprés muestra que el componente 1 explica el $40.8 \%$ de la variabilidad del incremento en altura de los últimos 2 años (Figura 7). Las variables que más contribuyen en ese componente son el IAF total de coihue, el IAF de coihue en el estrato superior, el IAF total del rodal y el área basal de coihue (Tabla 3). El componente 2 suma un $23 \%$ de variabilidad explicada (Figura 7) y las variables que más contribuyen son el IAF de ciprés en el estrato superior y el IAF total de ciprés. Para los renovales de coihue, el componente 1 explica el $40.4 \%$ de la variabilidad del incremento en altura de los últimos 2 años (Figura 7). Las variables que más contribuyen en ese componente son el IAF total de coihue, el IAF de coihue en

Tabla 3. Autovectores del análisis de componentes principales del incremento en altura de los últimos 2 años de los renovales de ciprés y coihue. IAF: índice de área foliar; AB: área basal.

Table 3. Eigenvectors of the principal components analysis of height increment of the last 2 years of cypress and coihue saplings. IAF: leaf area index; AB: basal area.

\begin{tabular}{lcccc}
\hline & Ciprés & & Coihue \\
\hline & & CP2 & CP1 & CP2 \\
IAF total & CP1 & 0.21 & $\mathbf{0 . 3 8}$ & 0.22 \\
IAF total ciprés & $\mathbf{0 . 3 8}$ & $\mathbf{0 . 5 0}$ & $\mathbf{0 . 5 0}$ \\
IAF ciprés est. sup. & 0.06 & $\mathbf{0 . 5 1}$ & -0.02 & $\mathbf{0 . 5 2}$ \\
IAF ciprés est. inf. & 0.04 & 0.03 & 0.10 & -0.01 \\
IAF total coihue & 0.08 & -0.14 & $\mathbf{0 . 4 0}$ & -0.14 \\
IAF coihue est. sup. & $\mathbf{0 . 3 9}$ & -0.11 & $\mathbf{0 . 4 0}$ & -0.10 \\
IAF coihue est. inf. & $\mathbf{0 . 3 9}$ & -0.16 & 0.11 & -0.19 \\
AB ciprés & 0.10 & 0.41 & 0.09 & 0.42 \\
AB coihue & 0.12 & 0.02 & $\mathbf{0 . 3 7}$ & 0.04 \\
AB total & $\mathbf{0 . 3 6}$ & 0.30 & 0.31 & 0.32 \\
Pendiente & 0.32 & -0.24 & 0.26 & -0.19 \\
Exposición & 0.28 & -0.25 & 0.31 & -0.21 \\
Altitud & 0.28 & 0.10 & -0.32 & 0.01 \\
\hline
\end{tabular}

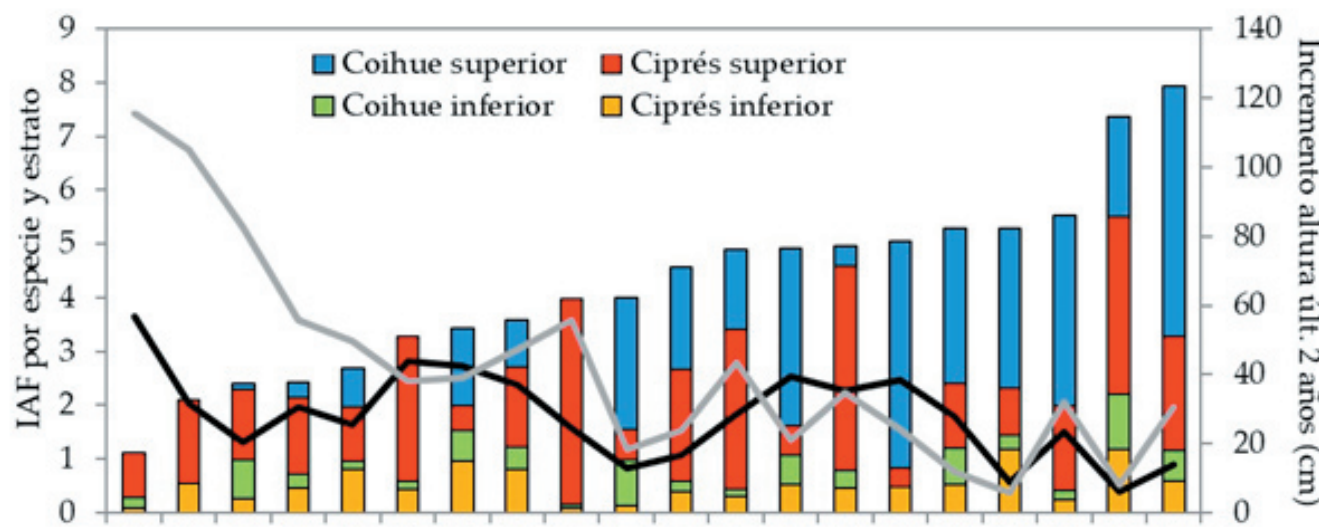

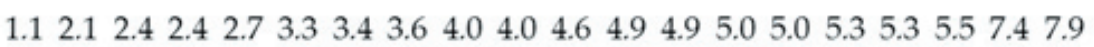

IAF del rodal

Figura 6. Incremento en altura de renovales de ciprés (línea negra) y de coihue (línea gris) bajo dosel con diferente participación de índice de área foliar (IAF) en el estrato superior e inferior de ciprés (barras rojas y naranjas) y en el estrato superior e inferior de coihue (barras azules y verdes).

Figure 6. Height increment of cypress (black line) and coihue (gray line) saplings under canopy with different participation of leaf area index (IAF) in the upper and lower strata of cypress (red and orange bars) and coihue (blue and green bars). 
Ciprés

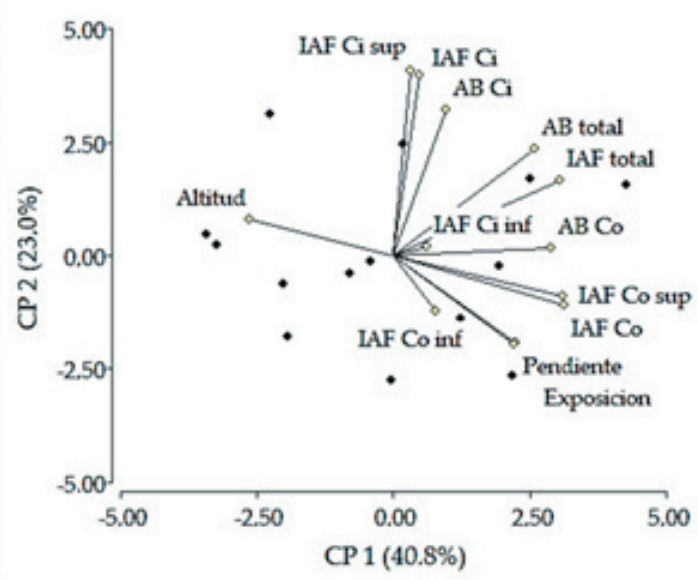

Coihue

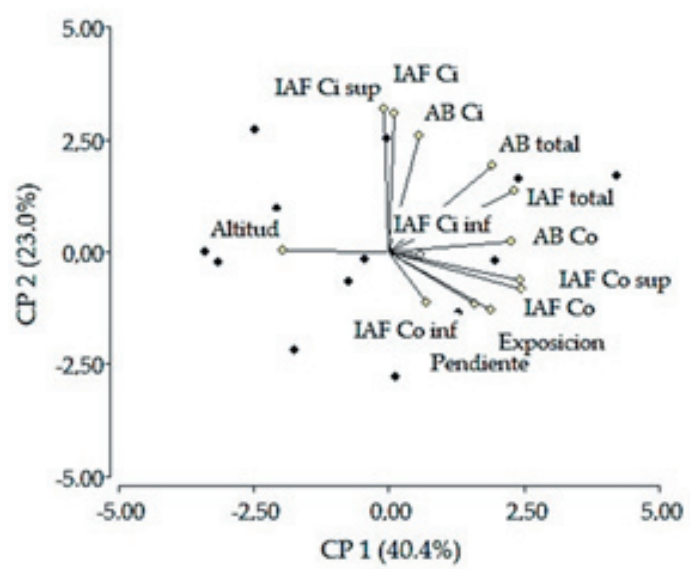

Figura 7. Análisis de componentes principales del incremento en altura de los últimos 2 años de los renovales de ciprés y coihue. IAF: índice de área foliar; AB: área basal; Ci: ciprés; Co: coihue; Sup: del estrato superior; Inf: del estrato inferior.

Figure 7. Principal components analysis of height increment of the last 2 years of cypress and coihue saplings. IAF: leaf área index; $\mathrm{AB}$ : basal area; $\mathrm{Ci}$ : cypress; $\mathrm{Co}$ : coihue; Sup: from the upper stratum; Inf: from the lower stratum.

el estrato superior, el IAF total del rodal y el área basal de coihue (Tabla 3). El segundo componente explica el $23.0 \%$ de la variabilidad y las variables que más contribuyen son el IAF de ciprés en el estrato superior y el IAF total de ciprés (Tabla 3 ).

Las parcelas que mostraron los mejores crecimientos de los renovales (i.e., con IAF del dosel menor a 4) tuvieron en promedio un área basal de $24.1 \mathrm{~m}^{2} / \mathrm{ha}(\mathrm{s}=2.9)$, con un estrato inferior con $4.3 \mathrm{~m}^{2} / \mathrm{ha}(\mathrm{s}=1.2)$ de ciprés y 1.5 $\mathrm{m}^{2} / \mathrm{ha}(\mathrm{s}=0.4$ ) de coihue, y un estrato superior con $13.5 \mathrm{~m}^{2} / \mathrm{ha}(\mathrm{s}=1.9)$ de ciprés y $7.9 \mathrm{~m}^{2} / \mathrm{ha}$ $(s=2.3)$ de coihue (Tabla 1). La pendiente y la exposición de estas parcelas fueron variables, con rangos entre $7 \%$ y $40 \%$ para la pendiente y entre $54^{\circ}$ y $260^{\circ}$ para la exposición.

\section{Discusión}

Del análisis del presente estudio surge que los renovales de ciprés y coihue presentan mayor crecimiento en altura cuando el IAF del dosel es bajo, pero ambas especies exhiben tendencias algo diferentes. Por un lado, el coihue presentaría una zona umbral de IAF del rodal para el desarrollo de la regeneración de alrededor de 4, por debajo del cual el incremento en altura aumenta de manera pronunciada, pudiendo alcanzar $60 \mathrm{~cm} / a$ ño. Por su parte, el incremento en altura del ciprés aumenta de manera gradual a medida que se reduce el IAF del rodal, mostrando una relación más débil entre ambas variables.
Estos resultados coinciden con lo hallado en la misma zona de estudio por Palenzuela y colaboradores (2018), quienes mostraron que existen mayores tasas de crecimiento de coihue cuando existe una reducción de la cobertura del dosel, mientras que la reacción en crecimiento del ciprés ante cambios en el dosel es más discreta. Dado que el IAF del rodal y la luz que llega a la regeneración tienen una relación asintótica inversa (Waring 1983; O'Hara et al. 2007; Comeau et al. 2009), las diferencias en el comportamiento de las especies bajo estudio son las esperables según su afinidad por la luz, siendo el ciprés la más tolerante a la sombra (Veblen and Lorenz 1987; Veblen 1989; Kitzberger et al. 2000). Esto implica que para que los renovales de ciprés puedan mostrar mayor crecimiento podrían necesitar reducciones fuertes de la cobertura del dosel (Arturi et al. 2001), o bien cambios en la competencia más cercana (del sotobosque). En este último sentido, Letourneau (2006) determinó que la liberación mediante la remoción de los arbustos en los alrededores de renovales de ciprés creciendo en un matorral provocó un aumento de las tasas de crecimiento, mostrando la capacidad de reacción del ciprés frente a un mayor espacio de crecimiento disponible. Sin embargo, es necesario tener en cuenta que reducciones fuertes en la cobertura pueden estimular el crecimiento y disminuir la capacidad de protección del dosel, que es un factor importante para la supervivencia de estas especies en los estadios iniciales, en 
particular en sitios expuestos a déficit hídrico (Gobbi and Schlichter 1998; Caselli et al. 2019, 2021b). Por lo tanto, bajo condiciones de manejo, se deberá establecer un equilibrio entre las condiciones del rodal que permitan promover el crecimiento de los renovales y aquellas que permitan cubrir sus necesidades de protección, especialmente en los primeros años de establecimiento (Valladares et al. 2004).

Por otra parte, el comportamiento diferencial entre ciprés y coihue bajo distinta ocupación del espacio de crecimiento por el dosel también se puede explicar por la diferente tolerancia a la falta de humedad de las especies. Diversos autores mostraron que los rodales con doseles densos transpiran más agua, y con ello agotan la humedad del suelo más rápido que en áreas abiertas o con doseles poco densos (Breshears et al. 1997; Aussenac 2000; Peck et al. 2012; Scharenbroch and Bockheim 2007; von Arx et al. 2013), lo que limitaría la humedad disponible para la regeneración. Entones, en aquellos rodales con alto IAF es esperable que los renovales de coihue puedan ver limitado su desarrollo por falta humedad, como sugirieron Caselli et al. (2019); esta condición no afectaría en la misma magnitud al ciprés por su mayor tolerancia a la sequía (Veblen et al. 1996; Suárez and Kitzberger 2008; Scholz et al. 2014). Este comportamiento podría ser utilizado para regular la composición del rodal mixto en la etapa temprana (Stancioiu and O'Hara 2006a). Para favorecer la participación de ciprés en la regeneración y permitirle un crecimiento en altura equilibrado con el coihue, el IAF del rodal debería mantenerse relativamente alto $(>4)$, mientras que en aquellos sectores donde se desee liberar el crecimiento de la regeneración de coihue, el IAF debería ser bajo $(<4)$.

Los renovales de coihue muestran una disminución del incremento en altura con la edad, aunque, al mismo tiempo, este efecto pudo estar influenciado por la correlación positiva de la edad con el IAF del dosel. Una disminución del incremento en altura con la edad es importante para la planificación en la etapa de regeneración del rodal, lo cual también se observó en otros estudios. Clark y Clark (1999), y Lusk (2004) encontraron una inversión en los rankings de incremento en altura de especies de crecimiento inicial rápido demandantes de luz y de las tolerantes a la sombra a medida que aumentaba la edad, lo que coincide con los resultados de este estudio. Lusk (2004) atribuyó esta pérdida de las ventajas de crecimiento de las especies demandantes de luz por sobre las tolerantes a la menor asignación relativa de biomasa al área foliar que ocurre con la edad. Asimismo, dada la correlación positiva entre la edad de los renuevos de coihue y el IAF del rodal, los renovales más antiguos pueden estar creciendo menos, también debido al efecto del mayor IAF del rodal. Estos primeros resultados indicarían que los renovales de coihue deben ser liberados de la competencia del dosel a edades tempranas ( $<15$ años) para potenciar su crecimiento acelerado. Sin embargo, sería interesante ampliar la base de datos a otros sitios para comprender mejor las relaciones aquí encontradas. Debido a que el incremento en altura de los renovales de ciprés no está condicionado por la edad, relación que tampoco se ha mostrado en árboles adultos (Dezzotti and Sancholuz [1991]), pueden persistir bajo dosel largo tiempo con escaso crecimiento (Veblen and Lorenz 1987) sin necesidad de intervenciones silviculturales.

Los estudios que comparan el desarrollo de renovales bajo diferentes IAF del dosel son realmente escasos, pero en ellos se observan tendencias similares a las de este estudio. Comeau et al. (2009) mostraron en los bosques boreales de Canadá que el incremento en diámetro y en altura de los renovales de Picea glauca plantados bajo dosel de Populus tremuloides aumentó cuando el IAF del dosel fue menor a 2 . Un comportamiento similar se informó cuando se relacionó el incremento en altura (Stancioiu and O'Hara 2006a) y la eficiencia de crecimiento (Stancioiu and O'Hara 2006b) de la regeneración de Abies alba y Fagus sylvatica, ambas muy tolerantes a la sombra, en comparación con Picea abies, de tolerancia intermedia. O'Hara et al. (2007) mostraron un mayor desarrollo en altura de Sequoia sempervirens cuando el IAF se redujo mediante raleos a menos de 4. Martinez Pastur et al. (2011) mostraron para Nothofagus pumilio en el sur de la Patagonia que los renovales alcanzaron un mayor desarrollo altura cuando el IAF del dosel estuvo por debajo de 2. De manera similar, Magalhães et al. (2013) observaron menor crecimiento de renovales de cinco especies arbóreas del Amazonas cuando el IAF fue mayor. Asimismo, Kara and Topaçoðlu (2018) encontraron un mayor crecimiento en diámetro de cuello y altura de renovales de Pinus sylvestris a medida que el IAF fue menor. Estas respuestas se atribuyen principalmente a la menor disponibilidad de 
luz bajo el dosel cuando el canopeo es más denso.

El incremento en altura de los renovales de coihue y de ciprés parece estar influenciado no sólo por el total de ocupación del espacio de crecimiento por parte del dosel, sino también por la participación de cada especie en el espacio ocupado. Del ACP surge que los renovales de ciprés y coihue ven mayormente afectado su crecimiento en altura debido al IAF total de coihue y al IAF de coihue en el estrato superior. Por otro lado, el IAF de ciprés en el estrato superior también es una variable explicativa de importancia del crecimiento de los renovales, aunque de menor peso que las anteriores, ya que explica un porcentaje menor de la variabilidad. Estas diferencias en la influencia de la ocupación del espacio de crecimiento de las especies en el dosel se debería a la diferente incidencia que tienen las especies del dosel en la cantidad y el tipo de luz que dejan pasar hacia el sotobosque (Lieffers et al. 1999; Hart and Chen 2006; Barbier et al. 2008; Angelini et al. 2015). De esta manera, una mayor ocupación del coihue disminuiría la cantidad de luz que llega al sotobosque, en comparación con la luz que podría alcanzar el sotobosque si el dosel estuviera ocupado por ciprés, condicionando el crecimiento de la regeneración. En general, se considera que las especies demandantes de luz y las angiospermas dejan pasar más luz hacia el sotobosque que las especies más tolerantes y que las coníferas (Comnes et al. 2005; Angelini et al. 2015), lo cual no coincidiría con nuestros resultados. Sin embargo, también se observó que el arreglo espacial de las ramas y hojas juega un papel importante en la luz que pasa hacia el sotobosque (Falster and Westoby 2003; Barbier et al. 2008), de manera que aquellas especies que muestran una distribución de hojas con un ángulo más horizontal interceptan más luz que aquellas que tienen una distribución más vertical (Angelini et al. 2015). El coihue es reconocido por su disposición en estratos de sus ramas y hojas (Stecconi 2006), lo que le permitiría captar mayor cantidad de luz.

Por último, las variables de ubicación fisiográfica de los renovales, en este caso representadas por la pendiente, la exposición y la altitud, tuvieron influencia sobre el crecimiento en altura de los renovales de ciprés y coihue. Las primeras dos variables tuvieron una influencia similar en ambas especies, aunque para el coihue parece tener más importancia la exposición. Una menor pendiente favorecería el crecimiento, al igual que las exposiciones $\mathrm{S}-\mathrm{E}$, en contraposición con las N-O, lo que coincide con los resultados para Nothofagus obliqua obtenidos por Thiers y colaboradores (2008) y para N. pumilio por Schlatter (1994), en el centro y el sur de Chile. La relación entre la productividad forestal, la exposición y la pendiente fue explicada en el efecto conjunto del microclima, la humedad del suelo y el desarrollo del suelo (Carmean 1965). Por su lado, la altitud muestra una influencia importante en el crecimiento de ciprés y coihue, con menor crecimiento en las altitudes más bajas. En cierta manera, este último resultado es sorprendente, ya que diversos autores mostraron mayores tasas de crecimiento en altitudes más bajas en especies del género Nothofagus (Schlatter 1994; Donoso 1995; Cárdenas and Lusk 2002), aunque mayormente estas diferencias se atribuyen a una mayor temperatura y una duración más larga del período de la estación de crecimiento (Garrido and Lusk 2002). Sin embargo, también en este género se observó, en Nueva Zelanda, la tendencia contraria en sitios sujetos a estrés hídrico (Wardle 1984). Cabe destacar que pese a las diferencias y similitudes con los resultados de otros autores, el objetivo de este estudio no fue conocer la relación de la altitud y el crecimiento de los renovales, por lo que el rango de altitudes muestreados es acotado, lo que podría estar influenciando las tendencias observadas.

\section{Conclusiones y ReCOMEndaciones}

Para potenciar el crecimiento de la regeneración avanzada de coihue y ciprés en el bosque mixto podrían adoptarse dos estrategias que tienden a separar a la dominancia de las especies dentro del rodal, utilizando el IAF como herramienta para tomar decisiones silviculturales. En los sectores donde se desee promover al coihue se debe mantener el IAF del dosel por debajo de 4, liberando a la regeneración a temprana edad. Por otro lado, en los sectores donde se desee promover mayor participación de ciprés en la regeneración se debería limitar el crecimiento de los renovales de coihue, manteniendo el IAF del rodal por encima de 4. Para seguir aportando a la definición de técnicas de manejo de la regeneración en bosques mixtos de ciprés y coihue, futuras investigaciones deberían profundizar en los efectos sobre el incremento en altura de la regeneración bajo diferentes estructuras del dosel (composición de especies en los estratos de altura y densidad del rodal), ampliando la base de datos a otras 
ubicaciones geográficas, altitudes, pendientes y exposiciones, para captar fuentes de variación debidas al gradiente ambiental en el que crecen estas especies y que podrían afectar a las tendencias encontradas en este primer estudio.

\section{REFERENCIAS}

Amoroso, M. M., and B. C. Larson. 2010. Stand development patterns as a consequence of the mortality in Austrocedrus chilensis forests. For Ecol Manage 259:1981-1992. https://doi.org/10.1016/j.foreco.2010.02.009.

Angelini, A., P. Corona, F. Chianucci, and L. Portoghesi. 2015. Structural attributes of stand overstory and light under the canopy. Ann Silvic Res 39:23-31.

Arturi, M. F., J. J. Fernando, J. F. Goya, P. F. Yapura, and J. L. Frangi. 2001. Tendencias espaciales de la regeneración de Austrocedrus chilensis en relación con el dosel arbóreo. Ecol Aust 11:31-38.

Asner, G. P., J. M. O. Scurlock, and J. A. Hicke. 2003. Global synthesis of leaf area index observations. Glob Chang Biol 14:237-243.

Augspurger, C. K. 1984. Seedling survival of tropical tree species: interactions of dispersal distance, light-gaps, and pathogens. Ecol 65(6):1705-1712. https://doi.org/10.2307/1937766.

Aussenac, G. 2000. Interactions between forest stands and microclimate: ecophysiological aspects and consequences for silviculture. Ann For Sci 57:287-301. https://doi.org/10.1051/forest:2000119.

Barbier S., F. Gosselin, and P. Balandier. 2008. Influence of tree species on understory vegetation diversity and mechanisms involved-A critical review for temperate and boreal forests. For Ecol Manage 254:1-15. https://doi.org/ 10.1016/j.foreco.2007.09.038.

Bazzaz, F. A. 1979. The Physiological Ecology of Plant Succession. Ann Rev Ecol Syst 10:351-371. https://doi.org/10.1146/ annurev.es.10.110179.002031.

Breshears, D. D., P. M. Rich, F. J. Barnes, and K. Campbell. 1997. Overstory-imposed heterogeneity in solar radiation and soil moisture in a semiarid woodland. Ecol Appl 7:1201-1215. https://doi.org/10.1890/1051-0761(1997)007[1201: OIHISR]2.0.CO;2.

Brokaw, N. V. 1985. Treefalls, regrowth, and community structure in tropical forests. Pp. 53-69 in S. T. A. Pickett and P. S. White (eds.). The ecology of natural disturbance and patch dynamics. Academic Press, Orlando, Florida, USA. https://doi.org/10.1016/B978-0-12-554520-4.50009-5.

Brown, M. J., and G. G. Parker. 1994. Canopy light transmittance in a chronosequence of mixed species deciduous forests. Can J For Res 24:1694-1703. https://doi.org/10.1139/x94-219.

Canham, C. D. 1989. Different Respones to Gaps Among Shade-Tollerant Tree Species. Ecol 70:548-550. https://doi.org/ $10.2307 / 1940200$

Canham, C. D., and P. L. Marks. 1985. The response of woody plants to disturbance: patterns of establishment and growth. Pp. 197-216 in S. T. A. Pickett and P. S. White (eds.). The ecology of natural disturbance and patch dynamics. Academic Press, Orlando, Florida, USA. https://doi.org/10.1016/B978-0-12-554520-4.50016-2.

Cárdenas, C., and C. H. Lusk. 2002. Juvenile height growth rates and sorting of three Nothofagus species on an altitudinal gradient. Gayana Bot 59(1):21-25. https://doi.org/10.4067/S0717-66432002000100004.

Carmean, W. H. 1965. Black Oak Site Quality in Relation to Soil and Topography in Southeastern Ohio. Soil Sci Soc Am J 29(3):308. https://doi.org/10.2136/sssaj1965.03615995002900030024x.

Caselli, M., M. F. Urretavizcaya, G. A. Loguercio, and G. E. Defossé. 2019. Light and moisture conditions suitable for establishing andean cypress and coihue beech seedlings in patagonia: A nursery approach. For Sci 65:27-39. https: //doi.org/10.1093/forsci/fxy032.

Caselli, M., G. A. Loguercio, M. F. Urretavizcaya, and G. E. Defossé. 2020. Developing Silvicultural Tools for Managing Mixed Forest Structures in Patagonia. For Sci 66:119-129. https://doi.org/10.1093/forsci/fxz052.

Caselli, M., G. A. Loguercio, M. F. Urretavizcaya, and G. E. Defossé. 2021a. Stand level volume increment in relation to leaf area index of Austrocedrus chilensis and Nothofagus dombeyi mixed forests of Patagonia, Argentina. For Ecol Manage 494:119337. https://doi.org/10.1016/j.foreco.2021.119337.

Caselli, M., M. F. Urretavizcaya, G. A. Loguercio, L. Contardi, S. Gianolini, and G. E. Defossé. 2021b. Effects of canopy cover and neighboring vegetation on the early development of planted Austrocedrus chilensis and Nothofagus dombeyi in north Patagonian degraded forests. For Ecol Manag 479:118543. https://doi.org/10.1016/j.foreco.2020.118543.

Chauchard, L. M., and J. C Barnaba. 1986. Plan de Ordenación Cuartel de Loma del Medio- Río Azul. Comisión Mixta, convenio IFONA- Servicio Forestal Andino. El Bolsón, Río Negro.

CIEFAP-MAyDS. 2016. Actualización de la clasificación de tipos forestales y cobertura del suelo de

la región Bosque Andino Patagónico. Informe Final. CIEFAP. Esquel.

Clark, D. A., and D. B. Clark. 1999. Assessing the growth of tropical rain forest trees: Issues for forest modeling and management. Ecol Appl 9:981-997. https://doi.org/10.1890/1051-0761(1999)009[0981:ATGOTR]2.0.CO;2.

Colmet-daage, F., M. L. Lanciotti, and A. A. Marcolin. 1995. Importancia forestal de los suelos volcánicos de la Patagonia Norte y Central. INTA-SAGyP. Argentina. Pp. 27.

Comeau, P. G., C. N. Filipescu, R. Kabzems, and C. DeLong. 2009. Growth of white spruce underplanted beneath spaced and unspaced aspen stands in northeastern B.C.-10 year results. Elsevier. https:// doi.org/10.1016/ j.foreco.2008.11.023. 
Coomes, D. A., R. B. Allen, W. A. Bentley, L. E. Burrows, C. D. Canham, L. Fagan, D. M. Forsyth, A. Gaxiola-Alcantar, R. L. Parfitt, W. A. Ruscoe, D. A. Wardle, D. J. Wilson, and E. F. Wright. 2005. The hare, the tortoise and the crocodile: The ecology of angiosperm dominance, conifer persistence and fern filtering. J Ecol 93:918-935. https://doi.org/10.1111/ j.1365-2745.2005.01012.x.

Dezzotti, A. 1996. Austrocedrus chilensis and Nothofagus dombeyi stand development during secondary succession, in northwestern Patagonia, Argentina. For Ecol Manage 89:125-137. https://doi.org/10.1016/S0378-1127(96)03860-1.

Dezzotti, A., M. Rodríguez Arias, A. Parisi, R. Sbrancia, and D. Roat. 2004. Colonización y crecimiento de renovales de Nothofagus después de cortas selectivas de un rodal en la Patagonia, Argentina. For Syst 13:329-337.

Dezzotti, A., and L. Sancholuz. 1991. Los bosques de Austrocedrus chilensis en Argentina: ubicación, estructura y crecimiento. Bosque 12:43-52. https://doi.org/10.4206/bosque.1991.v12n2-04.

Di Rienzo, J., F. C Casanoves, M. Balzarini, L. González, M. Tablada, and C. Robledo. 2017. InfoStat versión 2017.

Donoso, C. 1981. Tipos forestales de los bosques nativos de Chile. CONAF/PNUD-FAO, Programa de Investigación y Desarrollo Forestal.

Donoso, C. 1995. Bosques templados de Chile y Argentina. Variación, Estructura y dinámica. 3르 edición. Ed. Universitaria, Santiago, Chile. Pp. 485.

Donoso, P. J., D. P. Soto, R. E. Coopman, and S. Rodríguez-Bertos. 2013. Respuesta temprana de plantaciones de Nothofagus dombeyi y Nothofagus alpina a la disponibilidad de luz y tamaño de claro en un bosque degradado en el centro sur de Los Andes de Chile. Bosque 34:23-32. https://doi.org/10.4067/S0717-92002013000100004.

Drever, C. R., and K. P. Lertzman. 2001. Light-growth responses of coastal Douglas-fir and western redcedar saplings under different regimes of soil moisture and nutrients. Can J For Res 31:2124-2133. https://doi.org/10.1139/x01-149.

Emborg, J. 1998. Understorey light conditions and regeneration with respect to the structural dynamics of a near-natural temperate deciduous forest in Denmark. For Ecol Manage 106:83-95. https://doi.org/10.1016/S0378-1127(97)002995.

Falster, D. S., and M. Westoby. 2003. Leaf size and angle vary widely across species: what consequences for light interception? New Phytol 158:509-525. https://doi.org/10.1046/j.1469-8137.2003.00765.x.

Finzi, A. C., and C. D. Canham. 2000. Sapling growth in response to light and nitrogen availability in a southern New England forest. For Ecol Manage 131:153-165. https://doi.org/10.1016/S0378-1127(99)00206-6.

Garrido, C. C., and C. H. Lusk. 2002. Juvenile height growth rates and sorting of three Nothofagus species on an altitudinal gradient. Gayana Bot 59(1):21-25. https://doi.org/10.4067/S0717-66432002000100004.

Givnish, T. 1988. Adaptation to sun and shade: a whole-plant perspective. Aust J Plant Physiol 15:63-92. https://doi.org/ 10.1071/PP9880063.

Gobbi, M., and T. Schlichter. 1998. Survival of Austrocedrus chilensis seedlings in relation to microsite conditions and forest thinning. For Ecol Manage 111:137-146. https://doi.org/10.1016/S0378-1127(98)00314-4.

Gratzer, G., A. Darabant, P. Chhetri, P. Rai, and O. Eckmüllner. 2004. Interspecific variation in the response of growth, crown morphology, and survivorship to light of six tree species in the conifer belt of the Bhutan Himalayas. Can J For Res 34:1093-1107. https://doi.org/10.1139/x03-281.

Grosfeld, J. 2001. Análisis de la variabilidad morfológica y arquitectura de Austrocedrus chilensis (D. Don) Pic. Serm. et Bizzarri, Fitzroya cupressoides (Molina) I. M. Johnst., Pilgerodendron uviferum (D. Don) Florin y Cupressus sempervirens L. (CUPRESSACEAE). Universidad Nacional de Comahue. https://doi.org/10.1016/S0764-4469(00)01289-0.

Hart, S. A., Chen H. Y. H. 2006 - Understory vegetation dynamics of North American boreal forests. CRC Crit Rev Plant Sci 25:381-397. https://doi.org/10.1080/07352680600819286.

Hoffmann, A. E. 1982. Flora silvestre de Chile. Vol. 2: Zona austral. Santiago, Chile.

Kara, F., E. F. Loewenstein, and D. G. Brockway. 2017. Effects of basal area on survival and growth of longleaf pine when practicing selection silviculture. For Syst 26(1):1-12. https://doi.org/10.5424/fs/2017261-10043.

Kara, F., and O. Topaçoðlu. 2018. Influence of stand density and canopy structure on the germination and growth of Scots pine (Pinus sylvestris L.) seedlings. Environ Monit Assess 190(12):1-10. https://doi.org/10.1007/s10661-018-7129-x.

Kitzberger, T., D. F. Steinaker, and T. T. Veblen. 2000. Effects of climatic variability on facilitation of tree establishment in northern Patagonia. Ecol 81:1914-1924. https://doi.org/10.1890/0012-9658(2000)081[1914:EOCVOF]2.0.CO;2.

Suárez, M. L., and T. Kitzberger. 2008. Recruitment patterns following a severe drought: long-term compositional shifts in Patagonian forests. Can J For Res 38:3002-3010. https://doi.org/10.1139/X08-149.

Letourneau, F. 2006. Estudio de las interacciones positivas y negativas sobre el crecimiento de Austrocedrus chilensis durante una etapa inicial de desarrollo, en un matorral sucesional mésico. Tesis doctoral. Universidad Nacional del Comahue. Pp. 182.

Letourneau, F. J., E. Andenmatten, and T. Schlichter. 2004. Effect of climatic conditions and tree size on Austrocedrus chilensis-shrub interactions in northern Patagonia. For Ecol Manage 191:29-38. https://doi.org/10.1016/ j.foreco.2003.11.002.

Lieberman, M., D. Lieberman, and R. Peralta. 1989. Forests are not just Swiss cheese: canopy stereogeometry of nongaps in tropical forests. Ecol 70(3):550-552. https://doi.org/10.2307/1940201.

Lieffers, V. J., C. Messier, K. J. Stadt, F. Gendron, and P. G. Comeau. 1999. Predicting and managing light in the understory of boreal forests. Can J For Res 29:796-811. https://doi.org/10.1139/x98-165.

Loguercio, G. A. 1997. Erhaltung der Baumart Austrocedrus chilensis (D. Don) et Boutelje durch nachhaltige Nutzung. Tesis. Doctoral, Fac. de Cs. Ftales de la Universidad de Munich. 
Loguercio, G. A., and M. Rajchenberg. 2004. El “mal del ciprés” y la silvicultura del ciprés de la cordillera. Pp. 1-18 in J. Frangi and A. D. Brown (eds.). Ecología y manejo de los bosques nativos de Argentina.

Loguercio, G. A., M. F. Urretavizcaya, M. Caselli, and G. E. Defossé. 2018. Propuestas silviculturales para el manejo de bosques de Austrocedrus chilensis sanos y afectados por el mal del ciprés de Argentina. Silvicultura en Bosques Nativos, OSU Oregón USA:111-128.

Loguercio, G. A., and H. Ivancich. 2019. Prueba piloto para evaluar Bosques secundarios post-fuego de la región del BAP para aportar a la mejora del Inventario Nacional de Gases de Efecto Invernadero (GEI). Informe Final. Esquel.

Long, J. N., and F. W. Smith. 1984. Relation between size and density in developing stands: A description and possible mechanisms. For Ecol Manage 7:191-206. https://doi.org/10.1016/0378-1127(84)90067-7.

Lusk, C. H. 2004. Leaf area and growth of juvenile temperate evergreens in low light: Species of contrasting shade tolerance change rank during ontogeny. Funct Ecol 18:820-828. https://doi.org/10.1111/j.0269-8463.2004.00897.x.

Magalhães, N. dos S., R. A. Marenco, and M. A. B. Camargo. 2014. Do soil fertilization and forest canopy foliage affect the growth and photosynthesis of Amazonian saplings? Scie Agric 71(1):58-65. https://doi.org/10.1590/S010390162014000100008.

Martinez Pastur, G., M. V. Lencinas, P. Peri, and M. Arena. 2007. Photosynthetic plasticity of Nothofagus pumilio seedlings to light intensity and soil moisture. For Ecol Manage 243(2):274-282. https://doi.org/10.1016/j.foreco.2007.03.034.

Martinez Pastur, G. J., P. L Peri, J. M Cellini, M. V. Lencinas, M. Barrera, and H. Ivancich. 2011. Canopy structure analysis for estimating forest regeneration dynamics and growth in Nothofagus pumilio forests. Ann For Sci 68(3): 587-594. https://doi.org/10.1007/s13595-011-0059-1.

Müller-Using, B., and F. Schlegel. 1980. The development of Chilean Nothofagus seedlings in a nursery shading experiment at the University of Valdivia. Allgemeine Forst- und Jagdzeitung 151:79-96.

Niinemets, Ü. 2010. A review of light interception in plant stands from leaf to canopy in different plant functional types and in species with varying shade tolerance. Ecol Res 25:693-714. https://doi.org/10.1007/s11284-010-0712-4.

O'Hara, K. 1988. Stand structure and growing space efficiency following thinning in an even-aged Douglas-fir stand. Can J For Res 18:859-866. https://doi.org/10.1139/x88-132.

O'Hara, K. L. 2015. Multiaged silviculture: managing for complex forest stand structures. Oxford University Press, USA. https://doi.org/10.1093/acprof:oso/9780198703068.001.0001.

O'Hara, K. L., and R. F. Gersonde. 2004. Stocking control concepts in uneven-aged silviculture. Forestry 77:131-143. https://doi.org/10.1093/forestry/77.2.131.

O’Hara, K. L., P. T. Stancioiu, and M. A. Spencer. 2007. Understory stump sprout development under variable canopy density and leaf area in coast redwood. For Ecol Manage 244:76-85. https://doi.org/10.1016/j.foreco.2007.03.062.

Oliver, C., and B. Larson. 1996. Forest Stand Dynamics, Update Edition. Yale School of the Environment Other Publications.

Pafundi, L., M. F. Urretavizcaya, and G. E. Defossé. 2014. improving survival and growth of planted Austrocedrus chilensis seedlings in disturbed patagonian forests of Argentina by managing understory vegetation. Environ Manage 54:1412-1420. https://doi.org/10.1007/s00267-014-0363-2.

Pafundi, L., M. F. Urretavizcaya, and G. E. Defossé. 2016. Micro-environmental changes induced by shape and size of forest openings: Effects on Austrocedrus chilensis and Nothofagus dombeyi seedlings performance in a Pinus contorta plantation of Patagonia, Argentina. For Syst 25(3):e075. https://doi.org/10.5424/fs/2016253-08971.

Palenzuela, S. L., M. M. Amoroso, and S. M. Bogino. 2018. Regeneration dynamics of Austrocedrus chilensis and Nothofagus dombeyi in declining forests. Bosque 39:333-345. https://doi.org/10.4067/S0717-92002018000200333.

Peck, J. E., E. K. Zenner, and B. Palik. 2012. Variation in microclimate and early growth of planted pines under dispersed and aggregated overstory retention in mature managed red pine in Minnesota. Can J For Res 42:279- 290. https: //doi.org/10.1139/x11-186.

Puntieri, J., E. Raffaele, P. Martínez, D. Barthélémy, and C. Brion. 1999. Morphological and architectural features of young Nothofagus pumilio (Poepp. and Endl.) Krasser (Fagaceae). Bot J Linn Soc 130:395-410. https://doi.org/10.1111/ j.1095-8339.1999.tb00529.x.

Rasband, W. 2018. ImageJ 1.52a. URL: imagej.nih.gov/ij. US National Institutes of

Health: Bethesda, MD, USA.

Rusch, V., and S. A. Varela. 2019. Bases para el manejo de bosques nativos con ganadería en Patagonia Norte : parte I. Buenos Aires: INTA Ediciones. Pp. 160. ISBN: 978-987-8333-17-5 (digital).

Scharenbroch, B. C., and J. G. Bockheim. 2007. Impacts of forest gaps on soil properties and processes in old growth northern hardwood-hemlock forests. Plant Soil 294(1):219-233. https://doi.org/10.1007/s11104-007-9248-y.

Schlatter, J. E. 1994. Requerimientos de sitio para lenga, Nothofagus pumilio (Poepp. et Endl.) Krasser. Bosque 15(2):3-10. https://doi.org/10.4206/bosque.1994.v15n2-01.

Scholz, F. G., S. J. Bucci, and G. Goldstein. 2014. Strong hydraulic segmentation and leaf senescence due to dehydration may trigger die-back in Nothofagus dombeyi under severe droughts: a comparison with the co-occurring Austrocedrus chilensis. Trees 28(5):1475-1487. https://doi.org/10.1007/s00468-014-1050-x.

Schuler, T. M., and F. W. Smith. 1988. Effect of species mix on size/density and leaf-area relations in Southwest pinyon/ juniper woodlands. For Ecol Manage 25:211-220. https://doi.org/10.1016/0378-1127(88)90088-6.

Shao, G. 1996. Potential impacts of climate change on a mixed broadleaved-Korean pine forest stand: A gap model approach. Clim Change 34:263-268. https://doi.org/10.1007/BF00224637. 
Soto, D. P., P. J. Donoso, D. Uteau, and A. Zúñiga-Feest. 2009. Environmental factors affect the spatial arrangement of survival and damage of outplanted Nothofagus dombeyi seedlings in the chilean andes. Interciencia 34:100-105.

Stancioiu, P. T., and K. L. O'Hara. 2006a. Regeneration growth in different light environments of mixed species, multiaged, mountainous forests of Romania. Eur J For Res 125:151-162. https://doi.org/10.1007/s10342-005-0069-3.

Stancioiu, P. T., and K. L. O'Hara. 2006b. Leaf area and growth efficiency of regeneration in mixed species, multiaged forests of the Romanian Carpathians. For Ecol Manage 222:55-66. https://doi.org/10.1016/j.foreco.2005.10.018.

Stecconi, M. 2006. Variabilidad arquitectural de especies nativas de Nothofagus de la Patagonia. PhD thesis, Universidad Nacional del Comahue, Argentina.

Thiers, O., V. Gerding, and E. Hildebrand. 2008. Renovales de Nothofagus obliqua en centro y sur de Chile: Factores de sitio relevantes para su productividad. Libro de actas de Eco Reuniones. Segunda Reunión sobre los Nothofagus en la Patagonia. Esquel, Chubut, Argentina. Pp. 255-260.

Urretavizcaya, M. F., and G. E. Defossé. 2013. Effects of nurse shrubs and tree shelters on the survival and growth of two Austrocedrus chilensis seedling types in a forest restoration trial in semiarid Patagonia, Argentina. Ann For Sci 70:21-30. https://doi.org/10.1007/s13595-012-0234-z.

Urretavizcaya, M. F., and G. E. Defossé. 2019. Restoration of burned and post-fire logged Austrocedrus chilensis stands in Patagonia: Effects of competition and environmental conditions on seedling survival and growth. Int J Wildland Fire 28:365-376. https://doi.org/10.1071/WF18154.

Valladares, F., I. Aranda, and D. Sánchez-Gómez. 2004. La luz como factor ecológico y evolutivo para las plantas y su interacción con el agua. Pp. 335-369 in Ecología del bosque mediterráneo en un mundo cambiante.

Veblen, T., and D. Lorenz. 1987. Post fire stand development of Austrocedrus - Nothofagus forest in Patagonia. Vegetatio 73:113-126.

Veblen, T. T. 1989. Tree regeneration responses to gap along a transandean gradient. Ecology 70(3):541-543. https: //doi.org/10.2307/1940197.

Veblen, T. T., J. J. Armesto, B. R. Burns, T. Kitzberger, A. Lara, B. León, and K. R. Young. 1995. The ecology of the conifers of southern South America. Ecology of the Southern Conifers. Melbourne. Victoria.

Veblen, T. T., T. Kitzberger, and R. Villalba. 2004. Nuevos paradigmas en ecología y su influencia sobre el conocimiento de la dinámica de los bosques del sur de Argentina y Chile. Pp. 1-48 in M. F. Arturi, J. L. Frangi and J. F. Goya (eds.). Ecología y manejo de bosques de Argentina. Editorial de la Universidad Nacional de La Plata, La Plata, Argentina. ISBN: 950-34-0307-3.

von Arx, G., E. G Pannatier, A. Thimonier, and M. Rebetez. 2013. Microclimate in forests with varying leaf area index and soil moisture: potential implications for seedling establishment in a changing climate. J Ecol 101(5):1201-1213. https://doi.org/10.1111/1365-2745.12121.

Wardle, J. 1984. The New Zealand Beeches. New Zealand Forest Service, Christchurch.

Waring, R. H. 1983. Estimating forest growth and efficiency in relation to canopy leaf area. Adv Ecol Res 13:327-354. https://doi.org/10.1016/S0065-2504(08)60111-7.

Whitmore, T. C. 1989. Canopy gaps and the two major groups of forest trees. Ecology 70(3):536-538. https://doi.org/ $10.2307 / 1940195$.

Weinberger, P., and C. Ramírez. 2001. Microclima y regeneración natural de Raulí, Roble y Coigüe (Nothofagus alpina, N. obliqua y N. dombeyi). Bosque 22:11-26. https://doi.org/10.4206/bosque.2001.v22n1-02. 